\title{
APC Overexpression Suppresses the Pyroptosis of Subarachnoid Hemorrhage Model Cells through Regulating NLRP3 Inflammasome Pathway
}

Ai Yan ( $\sim$ yanai71@126.com )

Huzhou Central Hospital

Xuyan Pan

Huzhou Central Hospital

Xianqiang Wen

Huzhou Central Hospital

Xiaohu Nie

Huzhou Central Hospital

Yuntao Li

Huzhou Central Hospital

Research

Keywords: subarachnoid hemorrhage, APC, NLRP3, pyroptosis, 3K3A-APC

Posted Date: June 1st, 2021

DOI: https://doi.org/10.21203/rs.3.rs-556018/v1

License: (9) This work is licensed under a Creative Commons Attribution 4.0 International License.

Read Full License 


\section{Abstract}

Subarachnoid hemorrhage (SAH) is a kind of hemorrhagic stroke with high mortality. Activated protein C (APC) was implicated to play a neuroprotective role in central nervous system diseases. However, its role in SAH remains unclear. Our study aims to investigate the role of APC and its regulatory mechanism in $\mathrm{SAH}$. The SAH rat model was constructed by internal carotid artery puncture. The SAH cell model was established by the application of oxygen hemoglobin. ELISA was performed to detect the level of cytokines. Flow cytometry was used to detect the population of pyroptosis cells. Neurological functions of rats were estimated using modified Garcia scoring and balance beam test. SAH hemorrhage was estimated using modified Sugawara's scoring. APC was significantly increased and NLRP3 was decreased in SAH rat model in a time-dependent manner. The application of APC recombinant protein 3K3A-APC could notably ameliorate SAH hemorrhage and improve neurological functions. Besides, 3K3AAPC could inhibit pyroptosis in a dose-dependent manner in SAH cell model. Moreover, the inhibition of NLRP3 could reverse the effects induced by the knockdown of APC. Our study revealed that APC could ameliorate SAH-induced EBI by suppressing pyroptosis via inhibiting NLRP3 inflammasome, which would provide a novel strategy for the treatment of SAH.

\section{Introduction}

Subarachnoid hemorrhage (SAH) is a kind of hemorrhagic stroke defined as the hemorrhage in subarachnoid space (Dupont, et al. 2010). It is often caused by brain injury or aneurysm rupture. SAH is classified into five grades and grade 5 indicates the worst clinical prognosis (Rosen and Macdonald 2005). The average mortality of SAH patients is about $40 \%-50 \%$ (Teunissen, et al. 1996). Nevertheless, a previous study has suggested that about $46 \%$ of patients who survive from SAH often suffer from cognitive impairment (Suarez, et al. 2006). Early brain injury (EBI) is termed as the brain injury that occurs within 72 hours of SAH (Cahill, et al. 2006). The abnormal elevation of intracranial pressure, reduced cerebral blood perfusion, and the development of brain edema was considered to be associated with SAH-induced EBI (Sehba and Bederson 2006). However, the molecular mechanism underlying SAHinduced $\mathrm{EBI}$ remains unknown.

Activated protein $\mathrm{C}$ (APC) is an activated form of zymogen that plays a crucial role in regulating multiple biological processes (Griffin, et al. 2018). APC has been implicated to be a neuroprotective factor and is able to exert anti-apoptotic effects (Mosnier and Griffin 2006). 3K3A-APC is an artificial protein made through the replacement of three lysine residues by 3 alanine residues, which reduces more than $90 \%$ of anticoagulant activity but retains the original biological activities (Mosnier, et al. 2004). The application of 3K3A-APC has been translated to the treatment of acute ischemic stroke (Griffin, et al. 2016). However, the role of APC and its derivate in SAH-induced EBI remains to be further investigated.

Excessive inflammation is proposed to be a major cause of SAH-induced EBI (Liu, et al. 2021). NLRP3 inflammasome is composed of NOD-like receptor (NLRP3), ASC, and an adaptor protein (PYCARD) (Xu, et al. 2019). It is activated in response to pathogens and induces the secretion of interleukin-1 $\beta$ (IL-1 $\beta$ ) and 
IL-18, which can lead to an inflammatory cascade and initiate the programmed cell death known as pyroptosis (Abbate, et al. 2008; Kantono and Guo 2017). Previous studies indicate that the activation of NLRP3 inflammasome is associated with inflammatory injury and neurological disorders in SAH (Cao, et al. 2017; Zhou, et al. 2017). Moreover, pyroptosis induced by NLRP3 inflammasome has been implicated to correlate with neuroinflammatory injury in SAH (Xu, et al. 2020). However, the role of NLRP3 inflammasome and pyroptosis in SAH-induced EBI is rarely reported.

A previous study has indicated that APC could avert ischemia-reperfusion injury through the inhibition of NLRP3 inflammasome (Nazir, et al. 2017). However, the correlation between APC and NLRP3 inflammasome in SAH-induced EBI remains unclear. Our study established SAH cell and rat model to explore the biological activities of APC and NLRP3 inflammasome in SAH-induced EBI, aiming to provide novel strategies for SAH treatment.

\section{Materials And Methods}

\section{Cell culture and construction of SAH model}

Rat PC12 cells were used in this study and cultured with RPMI-1640 medium supplemented with $10 \%$ heat-inactivated horse serum and $5 \%$ fetal bovine serum. Cells were treated with $10 \mathrm{mM}$ oxygen hemoglobin $(0 x y H b)$ to construct SAH cell model. BAY11-7082 (5 $\mu \mathrm{mol} / \mathrm{L} ; \mathrm{S} 2913$, Selleck), the inhibitor of NLRP3, was dissolved in PBS and added into culture medium.

\section{Quantitative real-time PCR}

Total RNA was extracted using TRIzol Reagent (Invitrogen, USA). The cDNA synthesis kit (Thermo, USA) was used for CDNA synthesis. The CDNA was subjected to the following procedures: $95^{\circ} \mathrm{C}$ for 10 minutes followed by 40 cycles of $95^{\circ} \mathrm{C}$ for 15 seconds, $60^{\circ} \mathrm{C}$ for 45 seconds. GAPDH was used as the reference gene. Gene expression was calculated by the $2^{-\triangle \triangle C t}$ method. All data represented three replicates. All primers were listed as follows: APC, F: CACCCCTTTCAGTTCAAGTAGC, R: AAGACCCAGAATGGCGTTTAG; NLRP3, F: ACCTCAACAGACGCTACACCC, R: GCTGCTCCCTGGAACACC; GAPDH, F:

CGGTCAGGTCATCACTATC, R: CAGGGCAGTAATCTCCTTC.

\section{Western blot}

Total protein of cells or brain tissues was obtained using RIPA lysis buffer with protease inhibitor Cocktail (MCE, China). The protein was separated on 10\% SDS-PAGE and transferred to a nitrocellulose membrane. After the application of primary antibodies and secondary antibodies, the protein content was detected by ECL. The primary antibodies used in this study were provided as follows: APC (ab40778, abcam), NLRP3 (ab263899, abcam), active Caspase-1 (ab74279, abcam), GSDMD-N (\#39754, CST), and $\beta$-actin (\#3700, CST). 


\section{Knockdown of APC}

Three short hairpin RNAs (shRNA) were designed targeting human APC (shAPC-1:

GCAUGAAACUGCCUCCCAUTT; shAPC-2: GCAGGAAGCCCAUGAACAATT; shAPC-3:

GCCACUGACAUAUCUUCAUTT. The negative control shRNA (shNC) was taken as the reference with the following sequence: UUCUCCGAACGUGUCACGUTT. In 48 hours prior to experiments, cells were transfected with shRNAs using Lipofectamine 2000 (Invitrogen).

\section{ELISA}

The levels of IL-1 $\beta$ and IL-18 were estimated using corresponding ELISA kits according to the protocol of the manufacturer. Briefly, IL-1 $\beta$ and IL-18 antibodies were applied at $37^{\circ} \mathrm{C}$ for $2 \mathrm{~h}$. After the rinse of scrubbing solution, the secondary antibody was applied. Then, the stop solution was added and the optical density at $450 \mathrm{~nm}$ was estimated.

\section{Estimation of cell pyroptosis}

The activated caspase-1 was estimated to reflect the level of cell pyroptosis using FAM-FLICA Caspase-1 Assay Kit (ImmunoChemistry Technologies, USA). In brief, cells were incubated with caspase-1 detection probe for $1 \mathrm{~h}$ in the dark. After the rinse to remove unconjugated FLICA reagent, cells were stained with propidium iodide (PI) for 20 min. Flow cytometry was used to define pyroptosis cells as double positive for cleaved caspase-1 and PI.

\section{Construction of SAH rat model}

The SAH rat model was constructed by internal carotid artery puncture. After anesthesia, the external carotid artery was ligated and cut off. A nylon thread was put through the external carotid artery into the intracranial part of the internal carotid artery. When the branch met the resistance, advance the nylon thread to puncture the blood vessel of the internal carotid artery, which could lead to SAH in rats. Then, 2 $\mathrm{mg} / \mathrm{kg}$ 3K3A-APC was intraperitoneally injected every $12 \mathrm{~h}$. After the intervention for $24 \mathrm{~h}$ and $72 \mathrm{~h}$, brain tissues were collected for pathological analysis. There were six rats at each time point in different groups.

\section{Neurological function score}

The scoring of neurological functions was estimated using modified Garcia scoring and balance beam test. Modified Garcia scoring was composed of spontaneous activity, the extension of four limbs, climbing on the metal mesh wall, and touch response and whisker response of both sides of the trunk. Each of the first three items was 0-3 points, and that of the latter three items was 1-3 points, and total 
scores were 3-18 points. Balance beam test was defined as rats walking on a wooden rod $(20 \mathrm{~cm}$ wide), with $0-4$ points.

\section{SAH hemorrhage score}

The scoring of SAH hemorrhage was estimated by modified Sugawara's scoring. The head of rats was decapitated under anesthesia, the basal cistern and Willis' circle were photographed. The basal cistern was divided into 6 parts, and the scores for each part are as follows: 0 stood for no SAH; 1 was defined as a small amount of SAH; 2 was classified as medium SAH with several distinguishable blood vessels; 3 was defined as a large amount of SAH without distinct blood vessels. The final score was the sum of the score of six parts, among which $0-7,8-12$, and $13-18$ points were defined as mild, moderate, and severe hemorrhage.

\section{Statistical analysis}

GraphPad Prism version 7.0 (CA, USA) was used for data analyses and visualization. Quantitative data were represented as mean \pm standard deviation for more than three samples. The difference between groups was estimated by Student's t-test or one-way ANOVA. The P-value $<0.05$ indicated statistical significance.

\section{Results}

\section{The alternation of APC and NLRP3 exhibited a time- dependent manner in SAH rat model}

To preliminarily explore the role of APC and NLRP3 in SAH, we detected the expression of APC and NLRP3 in SAH rat model at different time points. Results showed that the mRNA level of APC was significantly reduced whereas that of NLRP3 was significantly elevated in a time-dependent manner in SAH rat model $(p<0.05)($ Fig. $1 A)$. Further, we found that the protein level of APC was also significantly decreased whereas NLRP3, active caspase-1, and GSDMD-N were significantly increased in SAH rat model in a time-dependent manner (Fig. 1B). These results indicated that APC and NLRP3 were associated with the development of $\mathrm{SAH}$, and they exhibited reversed expression patterns in $\mathrm{SAH}$.

\section{APC recombinant protein 3K3A-APC ameliorated the SAH induced injury in rat model}

To further explore the function of APC in SAH, we applied a recombinant APC protein termed 3K3A-APC in $\mathrm{SAH}$ rat model. After the intervention for $24 \mathrm{~h}$ or $72 \mathrm{~h}, 3 \mathrm{~K} 3 \mathrm{~A}-\mathrm{APC}$ significantly reduced SAH hemorrhage in rats $(p<0.05)$ (Fig. 2A-B). Besides, 3K3A-APC could significantly improve neurological functions which were significantly impaired in SAH model $(p<0.05)($ Fig. $2 C)$. Meanwhile, the balance beam score was 
significantly reduced after the application of 3K3A-APC $(p<0.05)$ (Fig. 2D). Then we collected brain tissues from rats and found that the level of IL-1 $\beta$ and IL-18 was significantly elevated in SAH rats whereas 3K3A-APC could significantly reduce their concentrations $(p<0.05)$ (Fig. 2E). Western blot revealed that the expression of NLRP3, active caspase-1, and GSDMD-N was significantly reduced after the intervention of 3K3A-APC (Fig. 2F). These results suggested that recombinant APC protein 3K3A-APC could ameliorate the hemorrhage and improve neurological functions in $\mathrm{SAH}$ through the suppression of pyroptosis.

\section{APC recombinant protein $3 \mathrm{~K} 3 \mathrm{~A}-\mathrm{APC}$ inhibited pyroptosis in a dose-dependent manner in SAH cell model}

Then we investigated the correlation between 3K3A-APC and pyroptosis in SAH cell model. The construction of SAH cell model was performed by the application of $10 \mathrm{mM} \mathrm{OxyHb}$, in which the application of $5,10,20 \mathrm{ng} / \mathrm{ml} 3 \mathrm{~K} 3 \mathrm{~A}-\mathrm{APC}$ significantly reduced the proportion of pyroptosis cells that were characterized by PI-positive and activate caspase-1-positive in a dose-dependent manner $(p<0.05)$ (Fig. 3A). Besides, the level of IL-1 $\beta$ and IL-18 was significantly inhibited after the application of 5, 10, 20 $\mathrm{ng} / \mathrm{ml} 3 K 3 \mathrm{~A}-\mathrm{APC}$, in which $20 \mathrm{ng} / \mathrm{ml}$ dose exhibited the highest efficiency $(\mathrm{p}<0.05)$ (Fig. 3B). Moreover, the protein level of NLRP3, active caspase-1, and GSDMD-N was notably suppressed by 3K3A-APC in a dose-dependent manner (Fig. 3C). These results indicated that 3K3A-APC could inhibit pyroptosis in SAH cell model in a dose-dependent manner.

\section{NLRP3 inhibitor reversed the activities of 3K3A-APC in SAH cell model}

To further verified the correlation between 3K3A-APC and pyroptosis in SAH, we applied a specific inhibitor of NLRP3, BAY11-7082, to explore its effect on 3K3A-APC. Three specific shRNAs targeting APC were designed to inhibit the expression of APC at mRNA and protein levels $(p<0.05)$ (Figure S1). The inhibition of APC significantly promoted the proportion of pyroptosis cells whereas the application of BAY11-7082 could reverse the effects $(p<0.05)$ (Fig. 4A). Besides, the level of IL-1 $\beta$ and IL-18 could be significantly elevated by shAPC and suppressed by BAY11-7082 ( $<<0.05)$ (Fig. 4B). Moreover, the expression of active caspase- 1 and GSDMD-N was increased by shAPC and further decreased by BAY117082 (Fig. 4C). These findings suggested that the inhibition of APC could promote pyroptosis in SAH cell through the blockade of NLRP3.

\section{Discussion}

Because of the long-term neurocognitive impairment, the quality of life (QOL) of SAH patients remained unsatisfied. Our study revealed that the expression of APC was significantly reduced and that of NLRP3 was significantly elevated in SAH rat model in a time-dependent manner. The application of APC 
recombinant protein 3K3A-APC could notably ameliorate SAH hemorrhage and improve neurological functions via the inhibition of pyroptosis. Moreover, the specific inhibition of NLRP3 exerted similar effects with 3K3A-APC. Our study revealed that APC played a protective role in SAH-induced EBI and 3K3A-APC was a promising strategy for SAH treatment.

APC was first recognized for its anticoagulant activity through the inactivation of coagulation factors and subsequent prevention of thrombosis (Ohga, et al. 2013). Meanwhile, APC was implicated to exert antiinflammatory activity (Joyce, et al. 2001). In the central nervous system, APC played a neuroprotective role in various brain diseases such as ischemic stroke and traumatic brain injury (Zlokovic and Griffin 2011; Griffin, et al. 2016). APC could be transported through the blood-brain barrier (BBB) via contact with its receptors and stabilized endothelial cells of the BBB (Deane, et al. 2009). In this study, we constructed a SAH rat model and found that APC was significantly reduced in SAH in a time-dependent manner, which indicated a protective role of APC in SAH.

As an engineering product of APC, 3K3A-APC was synthesized using 3 alanine residues to replace three lysine residues, which averted its coagulative activity and maintained the biological activities (Mosnier, et al. 2004). Preclinical studies demonstrated that 3K3A-APC exerted promising neuroprotective activities in the treatment of ischemic stroke (Mosnier, et al. 2004; Mosnier, et al. 2007). In phase II clinical trial, acute stroke patients treated with 3K3A-APC had a decreased incidence of hemorrhage (Lyden, et al. 2019). Moreover, 3K3A-APC could prevent the deposit of amyloid- $\beta$ and diminish neuroinflammatory responses in mice (Lazic, et al. 2019). Therefore, 3K3A-APC was a promising therapy in brain disorders. In our study, the application of 3K3A-APC significantly ameliorated the hemorrhage and improved neurological functions in SAH rat model. Moreover, 3K3A-APC could inhibit pyroptosis in a dose-dependent manner, which might account for its protective role in $\mathrm{SAH}$. Our findings indicated that 3K3A-APC was a promising strategy for SAH treatment.

NLRP3 inflammasome has been implicated to play a critical role in SAH-induced EBI. Hu et al. reported that the application of a specific TGR5 agonist, INT-777, could significantly reduce neuroinflammation via inhibiting NLRP3 inflammasome (Hu, et al. 2021). Besides, the specific blockade of NLRP3 could alleviate SAH-induced EBI by suppressing inflammation (Luo, et al. 2019). Moreover, fluoxetine could enhance autophagy to ameliorate SAH-induced EBI via inhibition of NLRP3 inflammasome (Li, et al. 2017). Our study revealed that the expression of NLRP3 was significantly elevated in SAH rat model. The application of NLRP3 inhibitor, BAY11-7082, could suppress the pyroptosis and reverse the function of APC in SAH cell model. These results indicated that NLRP3 was involved in the neuroprotective activity of APC in SAH. Additionally, previous studies indicated that NLRP3 inflammasome could activate caspase- 1 and promote the secretion of proinflammatory cytokines (IL-1 $\beta$ and IL-18) (Kelley, et al. 2019). Pyroptosis, which was activated by IL-1 $\beta$ and IL-18, was implicated to induce neuroinflammation in SAH-induced EBI (Chen, et al. 2021). Our study showed that the application of 3K3A-APC and the inhibition of NLRP3 could significantly reduce the level of IL-1 $\beta$ and IL-18 as well as the proportion of pyroptosis cells in SAH rat and cell models. Therefore, our study indicated that the neuroprotective role of APC was mediated by the suppression of pyroptosis via inhibiting NLRP3 inflammasome. 


\section{Conclusion}

To sum up, our study revealed that APC could ameliorate SAH-induced EBI by suppressing pyroptosis via inhibiting NLRP3 inflammasome, which would provide a novel strategy for the treatment of SAH.

\section{Declarations}

\section{Ethics approval and consent to participate}

The animal assay that performed in present study is approved by the animal ethics committee of Huzhou Central Hospital, Affiliated Hospital of Huzhou Normal University.

\section{Consent for publication}

Not applicable

\section{Availability of data and materials}

The dataset(s) supporting the conclusions of this article is(are) included within the article (and its additional file(s))

\section{Competing interests}

All author declared no conflict of interest

\section{Funding}

This research was financially supported by the Medical Science and Technology Project of Zhejiang Province (NO. 2020KY302) and Huzhou General Science and Social Development Project Foundation (NO. 2019GY39).

\section{Authors' contributions}

Xuyan Pan performed the experiments and wrote the draft; Xianqiang Wen analyzed the data and edited diagrams. Xiaohu Nie and Yuntao Li help to technical assistance. All authors have contributed to read and agreed the final content of manuscript for submission. All authors declared that there was no conflict of interest, and ethics approval and written informed consent had been obtained. Moreover, all authors stated that the content has not been published or submitted for publication elsewhere. 


\section{Acknowledgements}

We sincerely acknowledged the support given by the Huzhou Central Hospital, Affiliated Hospital of Huzhou Normal University, Huzhou for present research. Ai Yan designed this project and revised the manuscript;

\section{References}

1. Abbate A, Salloum FN, Vecile E, Das A, Hoke NN, Straino S, Biondi-Zoccai GG, Houser JE, Qureshi IZ, Ownby ED, et al. 2008. Anakinra, a recombinant human interleukin-1 receptor antagonist, inhibits apoptosis in experimental acute myocardial infarction. Circulation 117:2670-2683.

2. Cahill J, Calvert JW, Zhang JH. 2006. Mechanisms of early brain injury after subarachnoid hemorrhage. J Cereb Blood Flow Metab 26:1341-1353.

3. Cao S, Shrestha S, Li J, Yu X, Chen J, Yan F, Ying G, Gu C, Wang L, Chen G. 2017. Melatonin-mediated mitophagy protects against early brain injury after subarachnoid hemorrhage through inhibition of NLRP3 inflammasome activation. Sci Rep 7:2417.

4. Chen J, Zhang C, Yan T, Yang L, Wang Y, Shi Z, Li M, Chen Q. 2021. Atorvastatin ameliorates early brain injury after subarachnoid hemorrhage via inhibition of pyroptosis and neuroinflammation. $J$ Cell Physiol.

5. Deane R, LaRue B, Sagare AP, Castellino FJ, Zhong Z, Zlokovic BV. 2009. Endothelial protein C receptor-assisted transport of activated protein $\mathrm{C}$ across the mouse blood-brain barrier. $\mathrm{J}$ Cereb Blood Flow Metab 29:25-33.

6. Dupont SA, Wijdicks EF, Lanzino G, Rabinstein AA. 2010. Aneurysmal subarachnoid hemorrhage: an overview for the practicing neurologist. Semin Neurol 30:545-554.

7. Griffin JH, Mosnier LO, Fernandez JA, Zlokovic BV. 2016. 2016 Scientific Sessions Sol Sherry Distinguished Lecturer in Thrombosis: Thrombotic Stroke: Neuroprotective Therapy by RecombinantActivated Protein C. Arterioscler Thromb Vasc Biol 36:2143-2151.

8. Griffin JH, Zlokovic BV, Mosnier LO. 2018. Activated protein C, protease activated receptor 1, and neuroprotection. Blood 132:159-169.

9. Hu X, Yan J, Huang L, Araujo C, Peng J, Gao L, Liu S, Tang J, Zuo G, Zhang JH. 2021. INT-777 attenuates NLRP3-ASC inflammasome-mediated neuroinflammation via TGR5/cAMP/PKA signaling pathway after subarachnoid hemorrhage in rats. Brain Behav Immun 91:587-600.

10. Joyce DE, Gelbert L, Ciaccia A, DeHoff B, Grinnell BW. 2001. Gene expression profile of antithrombotic protein $\mathrm{c}$ defines new mechanisms modulating inflammation and apoptosis. J Biol Chem 276:11199-11203.

11. Kantono M, Guo B. 2017. Inflammasomes and Cancer: The Dynamic Role of the Inflammasome in Tumor Development. Front Immunol 8:1132. 
12. Kelley N, Jeltema D, Duan Y, He Y. 2019. The NLRP3 Inflammasome: An Overview of Mechanisms of Activation and Regulation. Int J Mol Sci 20.

13. Lazic D, Sagare AP, Nikolakopoulou AM, Griffin JH, Vassar R, Zlokovic BV. 2019. 3K3A-activated protein C blocks amyloidogenic BACE1 pathway and improves functional outcome in mice. J Exp Med 216:279-293.

14. Li JR, Xu HZ, Nie S, Peng YC, Fan LF, Wang ZJ, Wu C, Yan F, Chen JY, Gu C, et al. 2017. Fluoxetineenhanced autophagy ameliorates early brain injury via inhibition of NLRP3 inflammasome activation following subrachnoid hemorrhage in rats. J Neuroinflammation 14:186.

15. Liu GJ, Tao T, Zhang XS, Lu Y, Wu LY, Gao YY, Wang H, Dai HB, Zhou Y, Zhuang Z, et al. 2021. Resolvin D1 Attenuates Innate Immune Reactions in Experimental Subarachnoid Hemorrhage Rat Model. Mol Neurobiol.

16. Luo Y, Lu J, Ruan W, Guo X, Chen S. 2019. MCC950 attenuated early brain injury by suppressing NLRP3 inflammasome after experimental SAH in rats. Brain Res Bull 146:320-326.

17. Lyden P, Pryor KE, Coffey CS, Cudkowicz M, Conwit R, Jadhav A, Sawyer RN, Jr., Claassen J, Adeoye O, Song S, et al. 2019. Final Results of the RHAPSODY Trial: A Multi-Center, Phase 2 Trial Using a Continual Reassessment Method to Determine the Safety and Tolerability of 3K3A-APC, A Recombinant Variant of Human Activated Protein C, in Combination with Tissue Plasminogen Activator, Mechanical Thrombectomy or both in Moderate to Severe Acute Ischemic Stroke. Ann Neurol 85:125-136.

18. Mosnier LO, Gale AJ, Yegneswaran S, Griffin JH. 2004. Activated protein C variants with normal cytoprotective but reduced anticoagulant activity. Blood 104:1740-1744.

19. Mosnier LO, Griffin JH. 2006. Protein C anticoagulant activity in relation to anti-inflammatory and anti-apoptotic activities. Front Biosci 11:2381-2399.

20. Mosnier LO, Yang XV, Griffin JH. 2007. Activated protein C mutant with minimal anticoagulant activity, normal cytoprotective activity, and preservation of thrombin activable fibrinolysis inhibitordependent cytoprotective functions. J Biol Chem 282:33022-33033.

21. Nazir S, Gadi I, Al-Dabet MM, Elwakiel A, Kohli S, Ghosh S, Manoharan J, Ranjan S, Bock F, BraunDullaeus RC, et al. 2017. Cytoprotective activated protein C averts NIrp3 inflammasome-induced ischemia-reperfusion injury via mTORC1 inhibition. Blood 130:2664-2677.

22. Ohga S, Ishiguro A, Takahashi Y, Shima M, Taki M, Kaneko M, Fukushima K, Kang D, Hara T, Japan Childhood Thrombophilia Study G. 2013. Protein C deficiency as the major cause of thrombophilias in childhood. Pediatr Int 55:267-271.

23. Rosen DS, Macdonald RL. 2005. Subarachnoid hemorrhage grading scales: a systematic review. Neurocrit Care 2:110-118.

24. Sehba FA, Bederson JB. 2006. Mechanisms of acute brain injury after subarachnoid hemorrhage. Neurol Res 28:381-398.

25. Suarez JI, Tarr RW, Selman WR. 2006. Aneurysmal subarachnoid hemorrhage. N Engl J Med 354:387-396. 
26. Teunissen LL, Rinkel GJ, Algra A, van Gijn J. 1996. Risk factors for subarachnoid hemorrhage: a systematic review. Stroke 27:544-549.

27. Xu P, Hong Y, Xie Y, Yuan K, Li J, Sun R, Zhang X, Shi X, Li R, Wu J, et al. 2020. TREM-1 Exacerbates Neuroinflammatory Injury via NLRP3 Inflammasome-Mediated Pyroptosis in Experimental Subarachnoid Hemorrhage. Transl Stroke Res.

28. Xu S, Li X, Liu Y, Xia Y, Chang R, Zhang C. 2019. Inflammasome inhibitors: promising therapeutic approaches against cancer. J Hematol Oncol 12:64.

29. Zhou K, Shi L, Wang Z, Zhou J, Manaenko A, Reis C, Chen S, Zhang J. 2017. RIP1-RIP3-DRP1 pathway regulates NLRP3 inflammasome activation following subarachnoid hemorrhage. Exp Neurol 295:116-124.

30. Zlokovic BV, Griffin JH. 2011. Cytoprotective protein C pathways and implications for stroke and neurological disorders. Trends Neurosci 34:198-209.

\section{Figures}

A

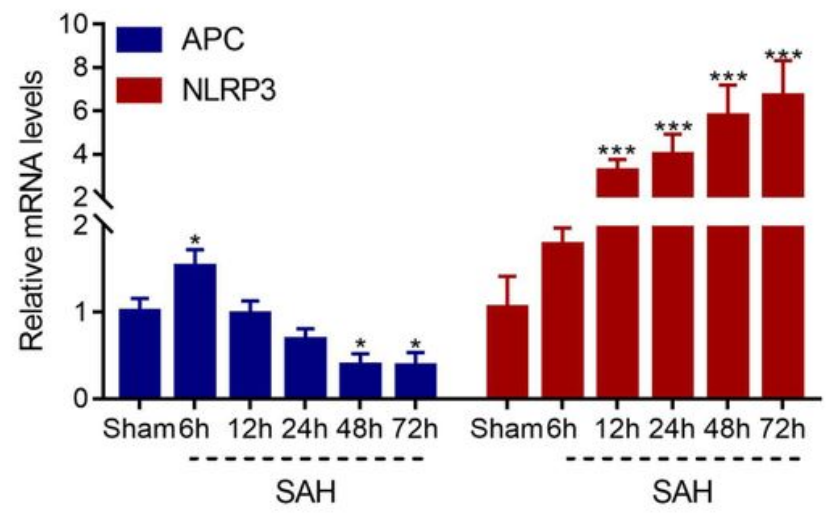

B

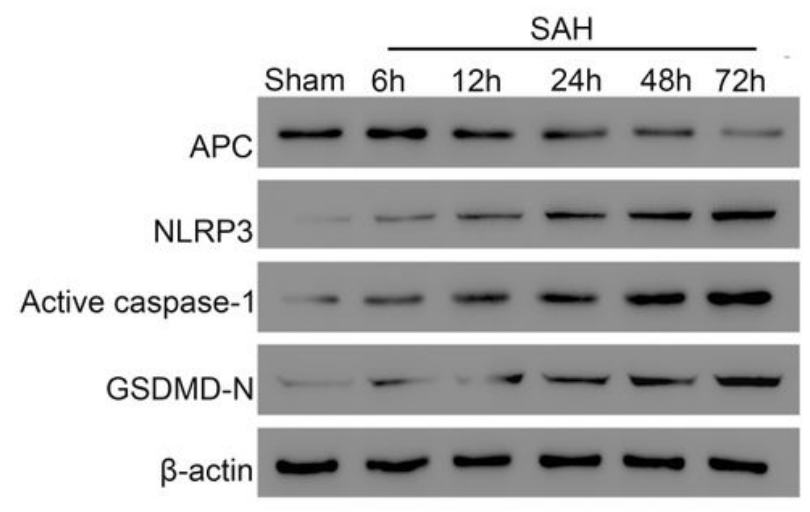

\section{Figure 1}

The expressions of APC and NLRP3 present a time-dependent manner in SAH model. A. The mRNA level of APC and NLRP3 in the brain of SAH rat model at $6 \mathrm{~h}, 12 \mathrm{~h}, 24 \mathrm{~h}, 48 \mathrm{~h}$ and $72 \mathrm{~h}$, respectively. B. The protein level of APC, NLRP3, active caspase-1 and GSDMD-N in the brain of SAH rat model at $6 \mathrm{~h}, 12 \mathrm{~h}, 24 \mathrm{~h}, 48 \mathrm{~h}$ and $72 \mathrm{~h}$, respectively. ${ }^{*} \mathrm{p}<0.05,{ }^{\star * \star} \mathrm{p}<0.001$. 
A

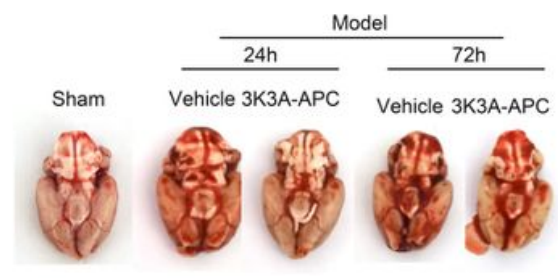

D

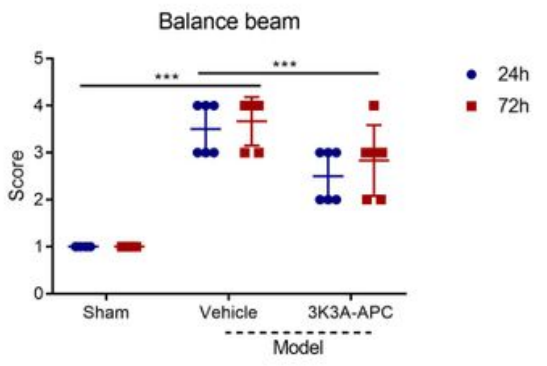

B

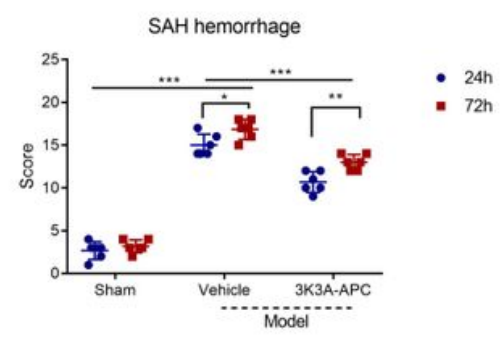

E

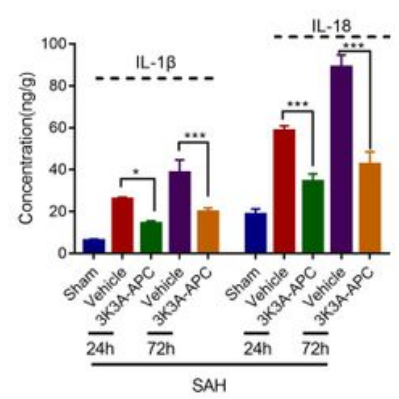

C

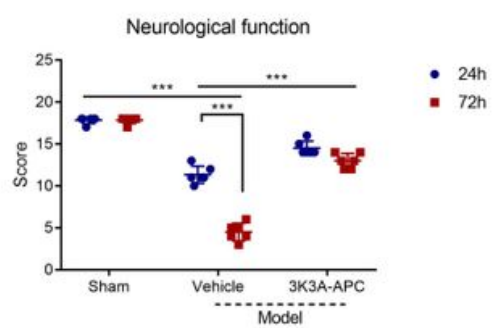

$\mathrm{F}$

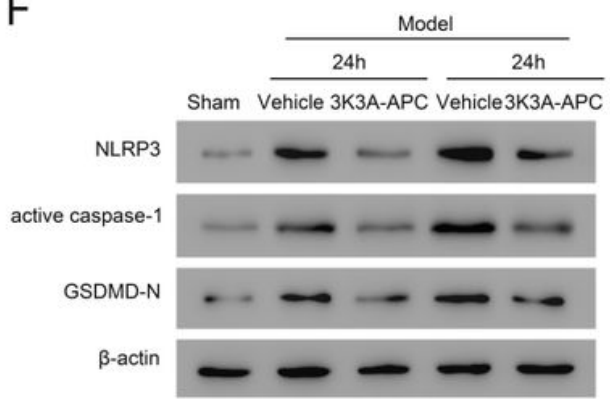

Figure 2

3K3A-APC ameliorated the SAH-induced injury in rat model. A. The morphology of brain tissues from SAH rat model treated with 3K3A-APC for $24 \mathrm{~h}$ and $72 \mathrm{~h}$. B-D. Hemorrhage score (B), neurological function score (C), and balance beam test score (D) of SAH rat model treated with 3K3A-APC for $24 \mathrm{~h}$ and $72 \mathrm{~h}$. E. The level of IL-1 $\beta$ and IL-18 of brain tissues from SAH rat model treated with 3K3A-APC for $24 \mathrm{~h}$ and $72 \mathrm{~h}$. F. The protein level of NLRP3, active caspase- 1 and GSDMD-N of brain tissues from SAH rat model treated with 3K3A-APC for $24 \mathrm{~h}$ and $72 \mathrm{~h} .{ }^{*} \mathrm{p}<0.0, * \star * \mathrm{p}<0.001$. 
A

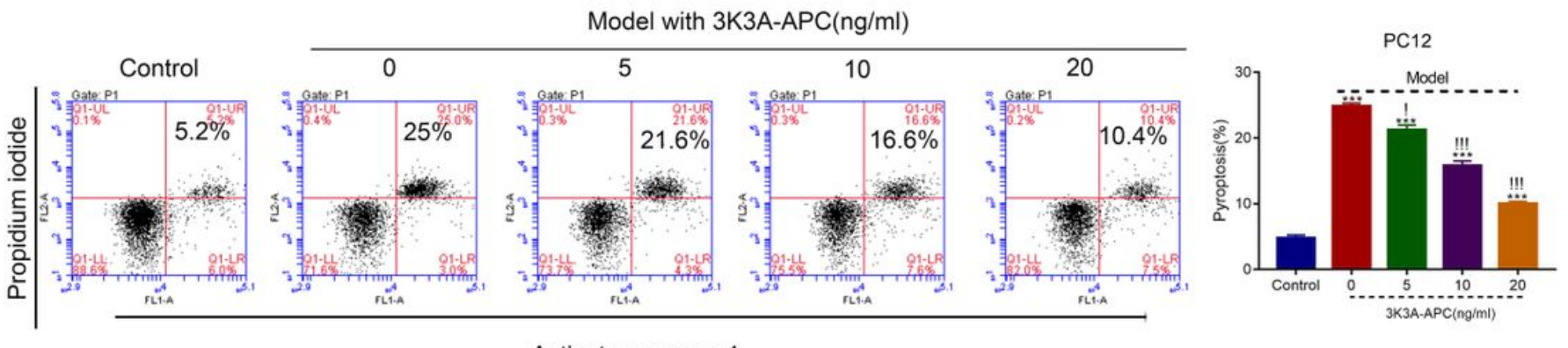

Activate caspase-1

$\mathrm{B}$

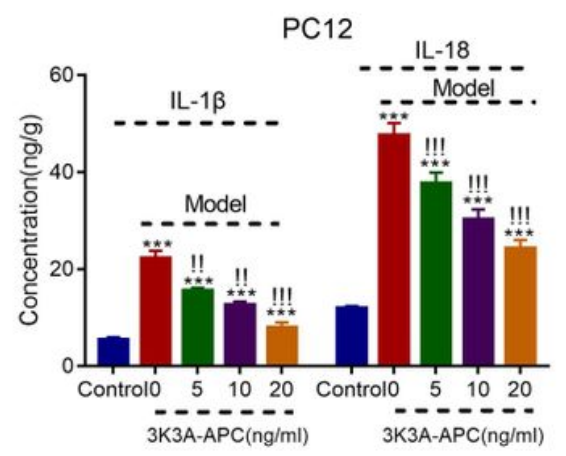

C

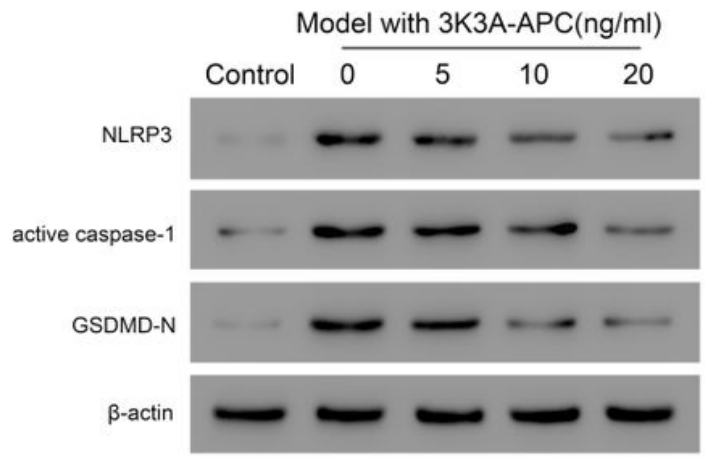

\section{Figure 3}

3K3A-APC inhibited the pyoptosis of SAH model cells in a dose-dependent manner. A. Flow cytometry revealed the population of pyroptosis cells treated with different concentrations of 3K3A-APC. B. The level of IL-1 $\beta$ and IL-18 in SAH model cells treated with different concentrations of 3K3A-APC. C. The protein level of NLRP3, active caspase-1 and GSDMD-N in SAH model cells treated with different concentrations of 3K3A-APC. *** $p<0.001$ vs control, !! $p<0.01 \mathrm{vs} 0 \mathrm{ng} / \mathrm{ml}$, !! $p<0.001 \mathrm{vs} 0 \mathrm{ng} / \mathrm{ml}$. 
A
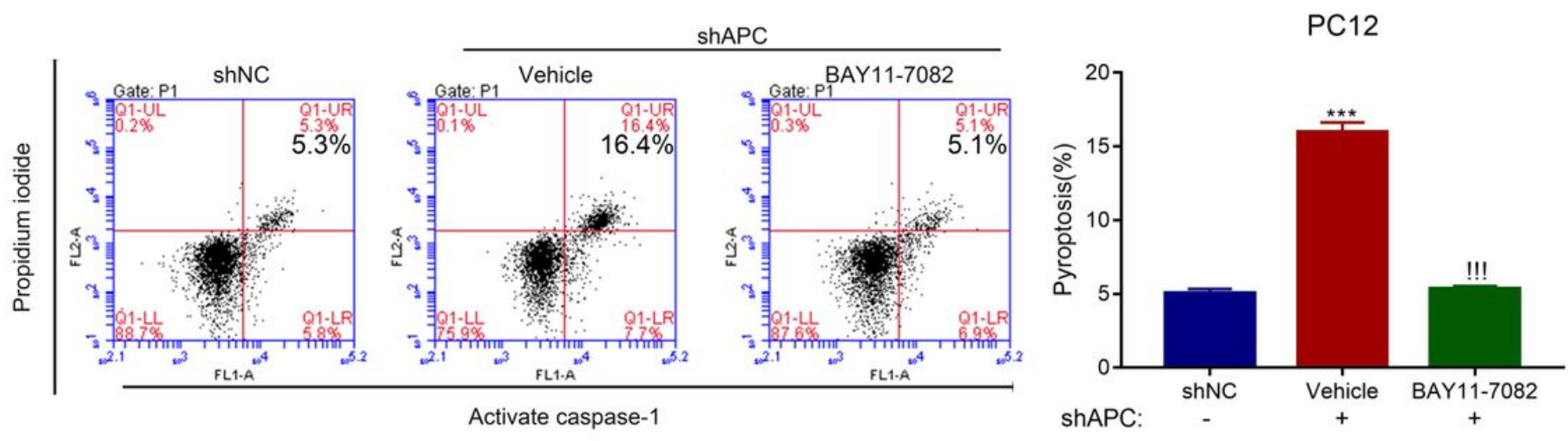

B

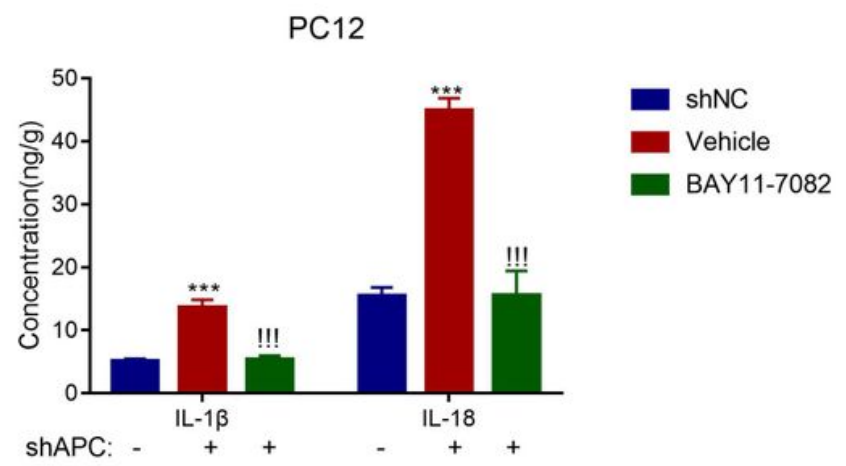

C

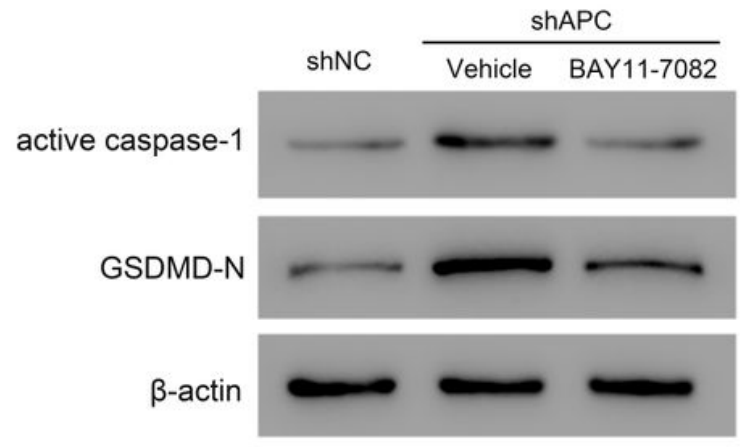

\section{Figure 4}

BAY11-7082 rescued the activities of APC inhibition in rat PC12 cells. A. Flow cytometry revealed the population of pyroptosis cells in PC12 cells applied with BAY11-7082 and shAPC. B. The level of IL-1 $\beta$ and IL-18 in cells applied with shAPC and BAY11-7082. C. The protein level of active caspase-1 and GSDMD-N in cells applied with shAPC and BAY11-7082. *** $p<0.001$ vs shNC, !! $p<0.001$ vs shAPC+ Vehicle.

\section{Supplementary Files}

This is a list of supplementary files associated with this preprint. Click to download.

- FigureS1.jpg 